Published in final edited form as:

Endocrinol Metab Clin North Am. 2019 December ; 48(4): 765-778. doi:10.1016/j.ecl.2019.08.007.

\title{
Renovascular Hypertension
}

\author{
Sandra M Herrmann, MD ${ }^{1}$ [Assistant Professor of Medicine], Stephen C Textor, MD ${ }^{1}$ \\ [Professor of Medicine] \\ ${ }^{1}$ Division of Nephrology and Hypertension, Mayo Clinic, Rochester, Minnesota, USA.
}

\begin{abstract}
Renovascular disease (RVD) is a major cause of secondary hypertension. Atherosclerotic renal artery stenosis is the most common type of RVD followed by fibromuscular dysplasia. It has long been recognized as the prototype of angiotensin-dependent hypertension. However, the mechanisms underlying the physiopathology of hypertensive occlusive vascular renal disease are complex and distinction between the different etiologies of RVD should be made. In an era of neutral results of recent prospective clinical trials regarding the benefit of renal artery revascularization, recognition of these distinct types of RVD with different degrees of renal occlusive disease is important for management. Many patients with RVD will be managed medically, while others with high risk clinical presentations and potential organ failure benefit from revascularization in addition to medical therapy. The greatest challenge is to individualize and implement the best approach for each patient in the setting of widely different comorbidities.
\end{abstract}

\section{Keywords}

Renovascular disease; renal artery stenosis; ischemic nephropathy; hypertension; renin and angiotensin

\section{Introduction}

Renovascular disease is caused by progressive occlusive renal artery disease presenting with a myriad of symptoms ranging from renovascular hypertension to ischemic nephropathy. Renovascular hypertension (RVH) is one of the most common causes of secondary hypertension and has been widely studied as the prototype of angiotensin-dependent hypertension. ${ }^{1}$ The great majority of the cases is caused by atherosclerotic lesions followed by fibromuscular dysplasia, but a variety of causes such renal artery dissection or embolic disease can produce the same symptoms. However, they are much less common.

Seminal studies performed by Goldblatt using 2-kidney 1-clip model in the early century established the role of hemodynamic hypoperfusion as a major cause of hypertension. ${ }^{2}$ Those studies were later corroborated by Page and Braun-Menendez identifying the vasoactive peptide angiotensin and its role of renin- angiotensin-aldosterone system (RAAS) axis activation in renovascular disease (RVD). ${ }^{3}$ These findings identified a potentially

Corresponding author: Address: 200 First Street SW, Rochester, MN, 55905, USA. herrmann.sandra@ mayo.edu. 
reversible cause of systemic elevated blood pressure. This was translated into clinical practice creating a potential therapy by surgical removal of the pressor kidney when renal artery occlusive disease was identified. ${ }^{4}$ This intervention did allow possible "cure" or better control of hypertension in an era when medical therapy for hypertension was not available. Unfortunately, surgical nephrectomy was often not successful in treating hypertension and was later discouraged. Intensive development of antihypertensive drugs led to discovery of multiple therapeutic classes including RAAS blockers in 1980's. This coincided with expansion of endovascular dilatation and stenting procedures.

While moderate renal artery stenosis (RAS) may be asymptomatic, hypertension eventually develops as RAS progresses. A commonly used criterion to identify hemodynamically significant stenosis is a decrease of at least $60 \%$ of the luminal diameter of the renal artery, which is associated with a peak systolic velocity $>200-300 \mathrm{~cm} / \mathrm{s}$ per Doppler ultrasonography. ${ }^{5,6}$ Nonetheless, studies have shown that the stenotic kidney is able to adapt to moderate flow reduction allowing relatively safe long-term use of antihypertensive drug therapy to control blood pressure. ${ }^{7}$ Over time, some renovascular occlusions progress and medical therapy alone fails to treat refractory hypertension. In these cases RVH can progress with the development of high-risk syndromes including hypertensive emergency associated with "flash pulmonary edema" and progressive decline of kidney function. ${ }^{8}$ At such a point, the combination of medical therapy plus revascularization of RAS may be required. In this review we will describe the epidemiology, types of RVD, pathophysiology, clinical features, diagnosis, and treatment recommendations for clinicians caring of complex cases in the current era.

\section{Epidemiology and Causes of Renovascular Hypertension}

Renovascular disease is a major cause of hypertension and it accounts for 1 to $5 \%$ of all cases of hypertension in the general population and $5.4 \%$ of secondary hypertension cases in young adults. ${ }^{9-11}$ RVD is more prevalent in the older population of $>65$ years of age when significant RAS (> than $60 \%$ of occlusion by Doppler ultrasound) may be nearly $7 \%{ }^{12}$ However, the real incidence and prevalence varies due to the variability of RAS definition and the type of the populations being studied. The prevalence may reach $40 \%$ in highly selected referral populations. ${ }^{13,14}$ In $90 \%$ of the cases, RVD is caused by atherosclerotic renal artery stenosis (ARAS) (Figure 1) followed by fibromuscular dysplasia (9\%). The remainder are due to miscellaneous causes as described in Table 1. ${ }^{15,16}$

\section{Atherosclerotic Renal Artery Stenosis}

Atherosclerotic renal artery stenosis is most common and is predominantly seen in older patients in the context of systemic atherosclerosis. Many of those plaques are extensions of aortic plaque into the renal artery. Hence, the location of atherosclerotic disease is usually near the origin of the artery, although it can be observed anywhere in the renal vessel. It can affect one or both renal arteries. Patients often have other associated risk factors such as diabetes, hypertension, smoking history, peripheral vascular disease, and coronary artery disease. It can vary from asymptomatic disease, discovered incidentally during imaging studies or other interventional procedures, to clinical syndromes that present with active 
cardiovascular symptoms including refractory hypertension, recurrent flash pulmonary edema, and progressive decline of renal function. $8,17,18$

Atherosclerotic RVD of some degree is found in up to $12-45 \%$ of the cases of patients undergoing vascular studies of patients with peripheral vascular disease and $14-40 \%$ of the cases of patients undergoing coronary angiography. ${ }^{13,19,20}$

\section{Fibromuscular Dysplasia}

Fibromuscular dysplasia (FMD) is a heterogeneous group of non-atherosclerotic and noninflammatory disease affecting intima or fibrous layers of the vascular wall. Commonly affects the renal and cerebral arteries (65-70\%) but can affect other vascular beds as well. 21,22 It can result in arterial stenosis, aneurysm formation with dissection and/or occlusion of medium-sized arteries. There are two subtypes: multifocal FMD, which has the appearance of a so-called "string of beads" and is the more common form; alternatively, focal FMD appears as a circumferential or tubular stenosis. FMD lesions are typically located away from the origin of the renal artery, often in the midportion of the vessel or at the first arterial bifurcation. It predominately affects women and is most commonly diagnosed in middle age. Interventional studies have suggested that among patients referred for renal revascularization for hypertension, FMD accounts for $16 \%$ or less. ${ }^{23}$ Hypertension is the most common presenting symptom, but other symptoms such as pulsatile tinnitus, stroke or chest pain may result from other affected vascular beds. ${ }^{24}$ Reports from arteriograms obtained from renal organ donors have showed presence of mild FMD in 3 to 5\% of those individuals without affecting renal blood flow or arterial pressure. ${ }^{17}$ Furthermore, a study using United States FMD Registry, showed that patients 65 years or older at the time of diagnosis of multifocal FMD were more likely to be asymptomatic and may have a more benign phenotype and fewer symptoms. ${ }^{25}$

In the prospective ARCADIA registry (Assessment of Renal and Cervical Artery Dysplasia), symptomatic patients with FMD underwent tomographic- or magnetic resonanceangiography from the aortic arch to the intracranial arteries, and those with cervical FMD from the diaphragm to the pelvis. Among patients with a cerebrovascular presentation, the prevalence of renal artery lesions was higher in patients with hypertension than in those without. Among patients with a renal presentation, the prevalence of cervical lesions was higher in patients with bilateral RAS than in those with unilateral renal artery lesions. These studies characterize FMD as a systemic arterial disease. ${ }^{26}$

\section{Pathophysiology of Renovascular Hypertension}

Renovascular hypertension is caused by reduction of blood flow perfusion to the kidney. Incidental RVD can be found in patients undergoing vascular imaging for other causes, but minor hemodynamic stenosis may not be of clinical significance. ${ }^{19,27}$ Studies using latex casts to characterize luminal occlusion fail to detect measurable pressure or flow changes at levels of RAS $<60 \%$ and suggest that the threshold for hemodynamic effects develops between 75 and $85 \%$ of luminal occlusion. ${ }^{28}$ Clinical studies show that occlusion using an expanded balloon causes renin release only after the pressure distal to the balloon falls by 
10-20\% below the pressure proximal to the lesion. ${ }^{29}$ These measurements correspond to a translesional peak systolic gradient of at least $20-25 \mathrm{mmHg}$ and luminal stenosis of at least $70 \% .{ }^{5}$ Hence, many lesions identified with clinical imaging tools are hemodynamically insignificant. As RAS progresses, the pressure and flow drop steeply (Figure 2). ${ }^{29}$ The development of hypertension in experimental RVD is directly related to renin release which raises systemic and distal renal pressures to maintain renal perfusion. The rise in blood pressure can be acute in experimental models using clip stenosis, or more gradual which is more typical of human RVD. This state of hypoperfusion triggers additional pathways that tend to restore kidney perfusion. It is well established from renovascular experimental models and in humans that activation of RAAS and sodium retention play a major role in $\mathrm{RVH}^{30,31}$

Renovascular occlusive disease results in release of renin from renal juxtaglomerular cells, which acts on its substrate angiotensinogen to produce angiotensin I. Angiotensin I is transformed by angiotensin-converting enzyme (ACE) into angiotensin II (A II) in the pulmonary capillary bed. Angiotensin II is a potent vasoconstrictor per se, and promotes the release of aldosterone from the adrenal cortex and retention of salt and water. ${ }^{32,33}$ The requirement for angiotensin in $\mathrm{RVH}$ was confirmed in a genetically modified angiotensin II subtype 1A receptor knockout (AT1A-/-) mouse model of 2-kidney 1-clip (2K1C) Goldblatt hypertension which did not develop hypertension. This study indicates the critical role of angiotensin II subtype 1A receptor in blood pressure control. ${ }^{34}$

It is important to point out that the role of the RAAS axis in RVD depends, in part, on whether or not a contralateral nonstenotic kidney is present. Unilateral RVH in human corresponds to the animal model of 2-kidney 1-clip (2K1C) Goldblatt model or also called "renin dependent" hypertension characterized by increased peripheral resistance. The rise in blood pressure stimulates pressure natriuresis by the intact contralateral kidney, which prevents volume expansion and sodium retention. Hence the nonstenotic kidney tends to counter the elevation of systemic blood pressure, which maintains reduced perfusion of the stenotic kidney and leads to continuous renin release. Although renovascular disease remains a prototype of angiotensin dependent hypertension, these hormonal responses are usually transient. As the occlusion progresses or if there is absence of a contralateral perfused kidney, the mechanisms of sustained hypertension differ. As intravascular volume increases due to impaired sodium and water excretion by an ineffective contralateral kidney, there is progressive decrease of renin secretion over time. This phase is referred to as "volume dependent" hypertension. ${ }^{35}$ Renin activity can be normal or low in this phase.

Glomerular filtration pressure is maintained distal to the stenosis by angiotensin II-mediated vasoconstriction preferentially acting on the efferent arterioles. This can reach a critical stage at which GFR requires angiotensin II as a result of reduced perfusion. Removal of the angiotensin II effect with blockade of the RAAS can dramatically drop GFR. Clinical examples for this include RAAS blockade with bilateral RAS or significant stenosis in a solitary functioning kidney. Administration of antihypertensive medications such as angiotensin-converting enzyme (ACE) inhibitors and angiotensin receptor blockers (ARBs) in patients with bilateral stenosis or solitary stenotic kidney must be done cautiously due to the potential worsening of renal function due to this mechanism. ${ }^{36}$ 
The pathophysiology of RVD has served as the basis for the use of radionuclide studies using captopril renography as well as renal vein renin measurements for the diagnosis and treatment of RAS. The RAAS axis has widespread effects beyond vasoconstriction and sodium retention. Angiotensin II has complex cellular interactions that lead to activation of inflammatory and fibrogenic mechanisms. These lead to vascular remodeling and tissue fibrosis within the stenotic kidney and left ventricular hypertrophy. ${ }^{37-39}$ Furthermore, ARAS typically occurs in the context of systemic atherosclerosis and the inflammatory milieu that accompanies this disease. Increased sympathetic activation, endothelial dysfunction, and increased oxidative stress have been also demonstrated in clinical studies. All these factors contribute to RVH in this population. ${ }^{40-42}$

The exact degree of vascular occlusion that threatens kidney function is still a matter of debate. The kidney is a highly perfused organ and capable of maintaining autoregulation of even with reduced arterial diameters. At some point, however, reduced renal perfusion beyond critical stenosis (75-80\%) leads to tissue hypoxia as measured by blood oxygen level-dependent (BOLD) imaging (Figure 3 ). ${ }^{7}$ One cannot exclude the possibility that atherosclerotic RVD represents a gradual process of repeated acute ischemic injury in the setting of an inflammatory milieu, leading to hypoxia and eventual loss of viable kidney function, designated "ischemic nephropathy". Identification of this population with decreased renal perfusion before this process becomes irreversible is an important clinical priority.

\section{Clinical Features}

As discussed above, RVH can develop in the setting of any cause of decreased blood flow to the affected kidney. FMD lesions most commonly affect midportion of the renal artery leading to early onset hypertension and affecting women preferentially between 15 and 55 years of age. It rarely causes major renal functional loss, although some progression may be seen in patients who develop vascular injury, such as dissection, but preferentially affects patients who are smokers. ${ }^{43}$ This type of renal artery lesion tends to respond well to percutaneous angioplasty.

Atherosclerotic renal artery stenosis is associated with more widespread deleterious effects, often involves both kidneys, and is a multi-organ disease process with a range of manifestations generally related to the severity of the vascular occlusion (Figure 4). ARAS is commonly encountered in the elderly population with other cardiovascular disease and associated comorbidities. Overall, these individuals have higher mortality compared to a normal age-adjusted population. ${ }^{44} \mathrm{~A}$ thorough physical examination including auscultation of abdominal/flank area in order to identify the presence of an abdominal bruit should be performed in all patients evaluated for hypertension. This may provide helpful hints to identify hypertensive patients with possible renal artery stenosis. If such a bruit is found, it should serve as added impetus for further imaging studies.

Many times the diagnosis of RAS occurs during imaging studies or procedures for other indications such as coronary catheterization, magnetic resonance (MR) or computed tomography (CT) angiography. The diagnosis of RVH in association with RAS is defined by 
a clinical syndrome with progressive elevation of blood pressure often leading to resistant or refractory hypertension with or without findings of chronic renal disease (Table 2). Clinical manifestations include progressive, sometimes rapid, increase in arterial pressure. This often occurs in elderly individuals with preexisting hypertension already treated with antihypertensive drugs. Therefore, clinicians should maintain a high suspicion for superimposed secondary causes of hypertension in a patient with progressively increase of antihypertensive drug requirements, especially in the setting of high risk for atherosclerotic disease. This is particularly important in individuals with a history of smoking which itself has a strong association with deleterious cardio-renal outcomes in patients with renovascular hypertension. ${ }^{45}$

Activation of the RAAS axis in this population magnifies blood pressure fluctuations especially if the patient is not on optimal medical therapy. Additional reabsorption of salt and water in the setting of increased RAAS activity commonly leads to volume overload and pulmonary congestion. The combination of volume excess and increased RAAS activation can accelerate target organ manifestations, including direct vascular injury, left ventricular hypertrophy, and renal dysfunction. These manifestations are seen more commonly in RVD than in patients with essential hypertension, if compared to age-matched population with same degree of blood pressure control. ${ }^{46}$

RVH in the setting of RVD can accelerate cardiovascular disease. When severe, the increase of blood pressure can be associated with volume retention and precipitate circulatory congestion associated with left ventricular dysfunction. This series of events has been designated "flash pulmonary edema" and represents a cardio-renal syndrome commonly associated with worsening renal function. ${ }^{47}$ Pulmonary edema associated with RVD leads to increased hospitalizations and increased morbidity and mortality in patients with congestive heart failure. ${ }^{48}$ Ultimately, progressive occlusive renal artery disease produces overt hypoxia within the kidney and irreversible worsening of kidney function designated "ischemic nephropathy" especially in the context of other risk factors such as diabetes, hypertension, and smoking. ${ }^{49}$

\section{Imaging and Laboratory Studies}

Presence of clinical manifestations that raise suspicion for RVD should prompt consideration of diagnostic imaging. However, before undertaking potentially costly and/or hazardous imaging procedures, clinicians should first establish the goals for the imaging study. Will this study change current medical management? Is the patient presenting with clinical features that warrant consideration for vascular intervention? Will the results of these studies lead to changes in medication, renal revascularization or even nephrectomy? The choice of imaging likely will depend on patient's clinical manifestations in the setting of current medical therapy. Advances in non-invasive imaging techniques have allowed greater diagnostic sensitivity and accuracy than ever before. Computed tomography angiography (CTA) and magnetic resonance angiography (MRA) and Doppler ultrasound are non-invasive imaging modalities commonly used to evaluate vasculature. However, none of these tests is perfect, and they are most informative for clinical purposes when the results are positive in a population at high risk for disease..$^{50,51}$ Duplex ultrasonography is often the 
first and least expensive study. There are no issues with IV contrast exposure. The limitations of this technique hinge upon its dependence upon operator skills and patient body habitus. If clinical suspicion for severe RVD is high and non-invasive tests remain inconclusive, catheter angiography may be performed. This procedure is usually reserved for patients considered candidates for endovascular intervention at the same session. One also can consider use of functional tests of renovascular disease using radionuclide renography with captopril when there is need to evaluate the relative function of each kidney prior to a therapeutic nephrectomy. 6,52 Mercapto-acetyltriglycine (MAG 3) is most commonly employed radionuclide due to its being more reliable in renal insufficiency. However, functional tests still have limited reliability in renal insufficiency, particularly in the setting of bilateral RVD. Hematologic and electrolytes tests will be normal or consistent with the degree of renal insufficiency. If creatinine progressively increases, this should warrant further evaluation including renal artery duplex ultrasound. Urinalysis is generally bland with minimal proteinuria. The presence of significant proteinuria or more active urinary sediment should raise concern for other renal parenchymal diseases.

Plasma renin activity levels alone are of limited value since they vary depending on the sodium and fluid retention status. ${ }^{53}$ The plasma aldosterone/renin ratio may be consistent with secondary aldosterone excess that may lead in some cases to hypokalemia. Measurement of renal vein renin levels has been used in the past for identification of overt lateralization of renin levels in the post-stenotic kidney and reduced in the contralateral kidney. These tests have been limited by the effects of medications and volume status. Because RVH is less commonly the primary indication to consider renal revascularization, as opposed to salvage and/or protection of renal function, these tests are rarely performed. They are most commonly used when contemplating therapeutic nephrectomy for blood pressure control.

\section{Management Strategies for RVD}

The treatment of RVH depends on the type of RVD and clinical manifestations. In patients with FMD, the renal artery may have multiple webs which are amenable to balloon angioplasty. Technical success rates may reach $90 \% .{ }^{54}$ Approximately $10-15 \%$ of these cases will develop restenosis, although repeat angioplasty can be performed. ${ }^{55}$ Despite successful technical outcomes, "cure" of hypertension with removal of all antihypertensive therapy happens in less than one third of the cases. The likelihood of "cure" depends on factors such as age of patient, duration and severity of hypertension. ${ }^{56}$ In general, clinicians should consider renovascular intervention in patients with FMD, especially for young patients with the goal of withdrawal or reducing the number of antihypertensive medications. ${ }^{57}$

The treatment of patients with ARAS differs somewhat from patients with FMD, since angioplasty alone commonly fails to maintain patency for proximal or ostial atherosclerotic lesions. Extensive calcified plaques at the vessel origin tend to recoil, leading to high rates of restenosis. ${ }^{58}$ After introduction of endovascular stents, rates of restenosis decreased, leading to technical success in nearly $100 \%$. Nonetheless, restenosis still can develops in up to $20 \%$ of cases for stents placed in vessels $<5 \mathrm{~mm}$ in diameter. ${ }^{59}$ 
Results of several randomized controlled trials comparing medical therapy to renal revascularization have failed to show definitive benefits of endovascular stent therapy in addition to medical therapy alone regarding improvement of blood pressure control, renal or cardiovascular outcomes. ${ }^{60-62}$ One can argue that contemporary therapies with multitargeted medical management of atherosclerosis, hypertension, and risk factors including smoking and diabetes control may have changed the natural history and clinical outcomes for many patients with atherosclerotic RVD. However, a major limitation of these studies reflects the fact that the population with "high risk" clinical presentations were underrepresented in these studies (i.e., rapidly progressive renal insufficiency, acute kidney injury during initiation of ACE/ARB therapy, intractable hypertension, and/or flash pulmonary edema). These would likely benefit from restoration of renal artery patency. Multiple observational studies indicate that such patients have major clinical benefits when renal circulation can be restored. The challenge for clinicians is to identify this subgroup of individuals with viable kidneys and intervene appropriately.

A clinical algorithm has been proposed for management of RVH and ischemic nephropathy (Figure 5). As with other vascular occlusive disease, identified patients with RVH should be followed to assure controlled hypertension and stable renal function. During follow up of 35 years with medical therapy in the trial populations, there was no evident advantage of revascularization, although individuals with normal urinary protein had evident cardiovascular and mortality benefits in a post-hoc analysis. ${ }^{63}$ Atherosclerotic disease commonly progresses over longer time period, and some individuals with other comorbidities develop further manifestations of high-grade stenosis and high risk features. For such individuals, clinicians need to periodically reevaluate risks versus benefits of revascularization of a potential salvageable kidney in order to prevent complete occlusion and/or irreversible injury.

\section{REFERENCES:}

1. Herrmann SM, Textor SC. Current Concepts in the Treatment of Renovascular Hypertension. American Journal of Hypertension 2018;31:139-49. [PubMed: 28985335]

2. Goldblatt H, Lynch J, Hanzal RF, Summerville WW. Studies on Experimental Hypertension: I. The Production of Persistent Elevation of Systolic Blood Pressure by Means of Renal Ischemia. The Journal of Experimental Medicine 1934;59:347-79. [PubMed: 19870251]

3. Basso N, Terragno NA. History about the discovery of the renin-angiotensin system. Hypertension (Dallas, Texas : 1979) 2001;38:1246-9.

4. Stanley JC. David M. Hume memorial lecture. Surgical treatment of renovascular hypertension. American Journal of Surgery 1997;174:102-10. [PubMed: 9293822]

5. Drieghe B, Madaric J, Sarno G, et al. Assessment of renal artery stenosis: side-by-side comparison of angiography and duplex ultrasound with pressure gradient measurements. European Heart Journal 2008;29:517-24. [PubMed: 18276621]

6. Hirsch AT, Haskal ZJ, Hertzer NR, et al. ACC/AHA 2005 Practice Guidelines for the management of patients with peripheral arterial disease (lower extremity, renal, mesenteric, and abdominal aortic): a collaborative report from the American Association for Vascular Surgery/Society for Vascular Surgery, Society for Cardiovascular Angiography and Interventions, Society for Vascular Medicine and Biology, Society of Interventional Radiology, and the ACC/AHA Task Force on Practice Guidelines (Writing Committee to Develop Guidelines for the Management of Patients With Peripheral Arterial Disease): endorsed by the American Association of Cardiovascular and Pulmonary Rehabilitation; National Heart, Lung, and Blood Institute; Society for Vascular Nursing; 
TransAtlantic Inter-Society Consensus; and Vascular Disease Foundation. Circulation 2006;113:e463-654. [PubMed: 16549646]

7. Gloviczki ML, Glockner JF, Crane JA, et al. Blood oxygen level-dependent magnetic resonance imaging identifies cortical hypoxia in severe renovascular disease. Hypertension (Dallas, Texas: 1979) 2011;58:1066-72.

8. Ritchie J, Green D, Chrysochou C, Chalmers N, Foley RN, Kalra PA. High-risk clinical presentations in atherosclerotic renovascular disease: prognosis and response to renal artery revascularization. American Journal of Kidney Diseases : the official journal of the National Kidney Foundation 2014;63:186-97. [PubMed: 24074824]

9. Olin JW, Piedmonte MR, Young JR, DeAnna S, Grubb M, Childs MB. The utility of duplex ultrasound scanning of the renal arteries for diagnosing significant renal artery stenosis. Annals of Internal Medicine 1995;122:833-8. [PubMed: 7741367]

10. Chrysochou C, Kalra PA. Epidemiology and natural history of atherosclerotic renovascular disease. Progress in Cardiovascular Diseases 2009;52:184-95. [PubMed: 19917329]

11. Noilhan C, Barigou M, Bieler L, Amar J, Chamontin B, Bouhanick B. Causes of secondary hypertension in the young population: A monocentric study. Annales de Cardiologie et d'Angeiologie 2016;65:159-64.

12. Hansen KJ, Edwards MS, Craven TE, et al. Prevalence of renovascular disease in the elderly: a population-based study. Journal of Vascular Surgery 2002;36:443-51. [PubMed: 12218965]

13. de Mast Q, Beutler JJ. The prevalence of atherosclerotic renal artery stenosis in risk groups: a systematic literature review. Journal of Hypertension 2009;27:1333-40. [PubMed: 19365285]

14. Piecha G, Wiecek A, Januszewicz A. Epidemiology and optimal management in patients with renal artery stenosis. Journal of Nephrology 2012;25:872-8. [PubMed: 23023722]

15. Safian RD, Textor SC. Renal-artery stenosis. The New England Journal of Medicine 2001;344:431-42. [PubMed: 11172181]

16. Lao D, Parasher PS, Cho KC, Yeghiazarians Y. Atherosclerotic renal artery stenosis--diagnosis and treatment. Mayo Clinic Proceedings 2011;86:649-57. [PubMed: 21719621]

17. Lorenz EC, Vrtiska TJ, Lieske JC, et al. Prevalence of renal artery and kidney abnormalities by computed tomography among healthy adults. Clinical Journal of the American Society of Nephrology : CJASN 2010;5:431-8. [PubMed: 20089492]

18. Rihal CS, Textor SC, Breen JF, et al. Incidental renal artery stenosis among a prospective cohort of hypertensive patients undergoing coronary angiography. Mayo Clinic Proceedings 2002;77:30916. [PubMed: 11936924]

19. Conlon PJ, O’Riordan E, Kalra PA. New insights into the epidemiologic and clinical manifestations of atherosclerotic renovascular disease. American Journal of Kidney Diseases : the official journal of the National Kidney Foundation 2000;35:573-87. [PubMed: 10739776]

20. Buller CE, Nogareda JG, Ramanathan K, et al. The profile of cardiac patients with renal artery stenosis. Journal of the American College of Cardiology 2004;43:1606-13. [PubMed: 15120819]

21. Sanidas EA, Seferou M, Papadopoulos DP, et al. Renal Fibromuscular Dysplasia: A Not So Common Entity of Secondary Hypertension. Journal of Clinical Hypertension (Greenwich, Conn) 2016;18:240-6.

22. Olin JW, Gornik HL, Bacharach JM, et al. Fibromuscular dysplasia: state of the science and critical unanswered questions: a scientific statement from the American Heart Association. Circulation 2014;129:1048-78. [PubMed: 24548843]

23. Krijnen P, van Jaarsveld BC, Steyerberg EW, Man in 't Veld AJ, Schalekamp MA, Habbema JD. A clinical prediction rule for renal artery stenosis. Annals of Internal Medicine 1998;129:705-11. [PubMed: 9841602]

24. Olin JW, Froehlich J, Gu X, et al. The United States Registry for Fibromuscular Dysplasia: results in the first 447 patients. Circulation 2012;125:3182-90. [PubMed: 22615343]

25. Bagh I, Olin JW, Froehlich JB, et al. Association of Multifocal Fibromuscular Dysplasia in Elderly Patients With a More Benign Clinical Phenotype: Data From the US Registry for Fibromuscular Dysplasia. JAMA Cardiology 2018;3:756-60. [PubMed: 29926082] 
26. Plouin PF, Baguet JP, Thony F, et al. High Prevalence of Multiple Arterial Bed Lesions in Patients With Fibromuscular Dysplasia: The ARCADIA Registry (Assessment of Renal and Cervical Artery Dysplasia). Hypertension (Dallas, Texas: 1979) 2017;70:652-8.

27. Garovic VD, Textor SC. Renovascular hypertension and ischemic nephropathy. Circulation 2005;112:1362-74. [PubMed: 16129817]

28. May AG, De Weese JA, Rob CG. Hemodynamic effects of arterial stenosis. Surgery 1963;53:51324. [PubMed: 13934072]

29. De Bruyne B, Manoharan G, Pijls NH, et al. Assessment of renal artery stenosis severity by pressure gradient measurements. Journal of American College of Cardiology 2006;48:1851-5.

30. Mueller CB, Surtshin A, Carlin MR, White HL. Glomerular and tubular influences on sodium and water excretion. The American Journal of Physiology 1951;165:411-22. [PubMed: 14838128]

31. Dustan HP. Physiologic consequences of renal arterial stenosis. The New England Journal of Medicine 1969;281:1348-54. [PubMed: 4901456]

32. Treadway KK, Slater EE. Renovascular hypertension. Annual Review of Medicine 1984;35:66592.

33. Sparks MA, Crowley SD, Gurley SB, Mirotsou M, Coffman TM. Classical Renin-Angiotensin system in kidney physiology. Comprehensive Physiology 2014;4:1201-28. [PubMed: 24944035]

34. Cervenka L, Horacek V, Vaneckova I, et al. Essential role of AT1A receptor in the development of 2K1C hypertension. Hypertension (Dallas, Texas: 1979) 2002;40:735-41.

35. Gavras H, Brunner HR, Thurston H, Laragh JH. Reciprocation of renin dependency with sodium volume dependency in renal hypertension. Science (New York, NY) 1975;188:1316-7.

36. Pickering TG. Renovascular hypertension: etiology and pathophysiology. Seminars in Nuclear Medicine 1989;19:79-88. [PubMed: 2652313]

37. Eirin A, Lerman LO. Darkness at the end of the tunnel: poststenotic kidney injury. Physiology (Bethesda, Md) 2013;28:245-53.

38. Gloviczki ML, Keddis MT, Garovic VD, et al. TGF expression and macrophage accumulation in atherosclerotic renal artery stenosis. Clinical journal of the American Society of Nephrology : CJASN 2013;8:546-53. [PubMed: 23258796]

39. Khangura KK, Eirin A, Kane GC, et al. Cardiac function in renovascular hypertensive patients with and without renal dysfunction. American Journal of Hypertension 2014;27:445-53. [PubMed: 24162729]

40. Johansson M, Elam M, Rundqvist B, et al. Increased sympathetic nerve activity in renovascular hypertension. Circulation 1999;99:2537-42. [PubMed: 10330385]

41. Lerman LO, Nath KA, Rodriguez-Porcel M, et al. Increased oxidative stress in experimental renovascular hypertension. Hypertension (Dallas, Texas: 1979) 2001;37:541-6.

42. Textor SC, Lerman LO. Paradigm Shifts in Atherosclerotic Renovascular Disease: Where Are We Now? Journal of the American Society of Nephrology: JASN 2015;26:2074-80. [PubMed: 25868641]

43. O'Connor S, Gornik HL, Froehlich JB, et al. Smoking and Adverse Outcomes in Fibromuscular Dysplasia: U.S. Registry Report. Journal of the American College of Cardiology 2016;67:1750-1. [PubMed: 27056782]

44. Vassallo D, Kalra PA. Progress in the treatment of atherosclerotic renovascular disease: the conceptual journey and the unanswered questions. Nephrology, dialysis, transplantation : official publication of the European Dialysis and Transplant Association - European Renal Association 2016;31:1595-605.

45. Drummond CA, Brewster PS, He W, et al. Cigarette smoking and cardio-renal events in patients with atherosclerotic renal artery stenosis. PloS One 2017;12:e0173562. [PubMed: 28306749]

46. Losito A, Fagugli RM, Zampi I, et al. Comparison of target organ damage in renovascular and essential hypertension. American Journal of Hypertension 1996;9:1062-7. [PubMed: 8931830]

47. Messerli FH, Bangalore S, Makani H, et al. Flash pulmonary oedema and bilateral renal artery stenosis: the Pickering Syndrome. European Heart Journal 2011;32:2231-5. [PubMed: 21406441]

48. Kane GC, Xu N, Mistrik E, Roubicek T, Stanson AW, Garovic VD. Renal artery revascularization improves heart failure control in patients with atherosclerotic renal artery stenosis. Nephrology, 
Dialysis, Transplantation : official publication of the European Dialysis and Transplant Association - European Renal Association 2010;25:813-20.

49. Textor SC, Lerman L. Renovascular hypertension and ischemic nephropathy. American Journal of Hypertension 2010;23:1159-69. [PubMed: 20864945]

50. Herrmann SM, Textor SC. Diagnostic criteria for renovascular disease: where are we now? Nephrology, Dialysis, Transplantation : official publication of the European Dialysis and Transplant Association - European Renal Association 2012;27:2657-63.

51. Textor SC. Pitfalls in imaging for renal artery stenosis. Annals of Internal Medicine 2004;141:7301. [PubMed: 15520432]

52. Fommei E, Ghione S, Hilson AJ, et al. Captopril radionuclide test in renovascular hypertension: a European multicentre study. European Multicentre Study Group. European Journal of Nuclear Medicine 1993;20:617-23. [PubMed: 8370384]

53. Wilcox CS. Use of angiotensin-converting-enzyme inhibitors for diagnosing renovascular hypertension. Kidney International 1993;44:1379-90. [PubMed: 8301939]

54. Tegtmeyer CJ, Selby JB, Hartwell GD, Ayers C, Tegtmeyer V. Results and complications of angioplasty in fibromuscular disease. Circulation 1991;83:I155-61. [PubMed: 1825043]

55. Surowiec SM, Sivamurthy N, Rhodes JM, et al. Percutaneous therapy for renal artery fibromuscular dysplasia. Annals of Vascular Surgery 2003;17:650-5. [PubMed: 14534846]

56. Bonelli FS, McKusick MA, Textor SC, et al. Renal artery angioplasty: technical results and clinical outcome in 320 patients. Mayo Clinic Proceedings 1995;70:1041-52. [PubMed: 7475333]

57. Davidson RA, Barri Y, Wilcox CS. Predictors of cure of hypertension in fibromuscular renovascular disease. American Journal of Kidney Diseases : the official journal of the National Kidney Foundation 1996;28:334-8. [PubMed: 8804230]

58. van de Ven PJ, Kaatee R, Beutler JJ, et al. Arterial stenting and balloon angioplasty in ostial atherosclerotic renovascular disease: a randomised trial. Lancet (London, England) 1999;353:2826.

59. Textor SC, Misra S, Oderich GS. Percutaneous revascularization for ischemic nephropathy: the past, present, and future. Kidney International 2013;83:28-40. [PubMed: 23151953]

60. Bax L, Woittiez AJ, Kouwenberg HJ, et al. Stent placement in patients with atherosclerotic renal artery stenosis and impaired renal function: a randomized trial. Annals of Internal Medicine 2009;150:840-8, w150-1. [PubMed: 19414832]

61. Wheatley K, Ives N, Gray R, et al. Revascularization versus medical therapy for renal-artery stenosis. The New England Journal of Medicine 2009;361:1953-62. [PubMed: 19907042]

62. Cooper CJ, Murphy TP, Cutlip DE, et al. Stenting and medical therapy for atherosclerotic renalartery stenosis. The New England Journal of Medicine 2014;370:13-22. [PubMed: 24245566]

63. Murphy TP, Cooper CJ, Pencina KM, et al. Relationship of Albuminuria and Renal Artery Stent Outcomes: Results From the CORAL Randomized Clinical Trial (Cardiovascular Outcomes With Renal Artery Lesions). Hypertension (Dallas, Tex : 1979) 2016;68:1145-52. 


\section{Key Points:}

- Renovascular disease is a major cause of secondary hypertension and may present with a range of clinical manifestations from asymptomatic hypertension to renal insufficiency and pulmonary congestion

- Distinction among types, degrees and clinical manifestations of renal artery stenosis should be made in order to define the best management for individual patients approach

- Results of prospective trials of optimal medical therapy indicate no additional benefits from renal artery stenting over the course of the trials for individuals with moderate renovascular disease

- Comprehensive medical therapy including use of agents that block the reninangiotensin system and lipid control should be the cornerstone for management atherosclerotic RVD

- Clinicians need to recognize renovascular hypertension progressing to "high risk" clinical presentations that should undergo renal revascularization to achieve better renal and cardiovascular outcomes 


\section{Atherosclerotic Renovascular Disease}

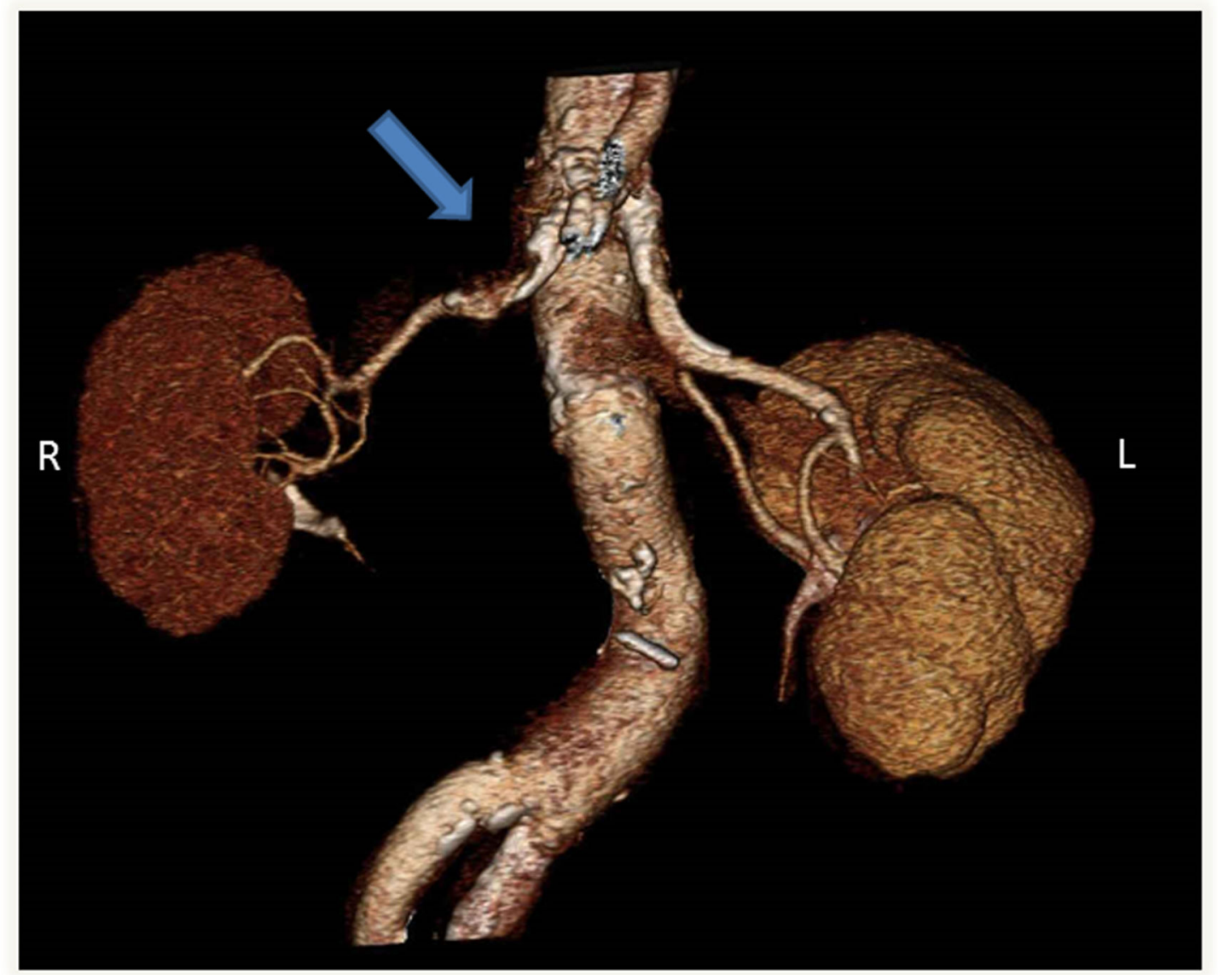

Figure 1:

$\mathrm{CT}$ angiography depicting moderate atherosclerotic renovascular disease affecting the right kidney (arrow). Right (R); Left (L) 


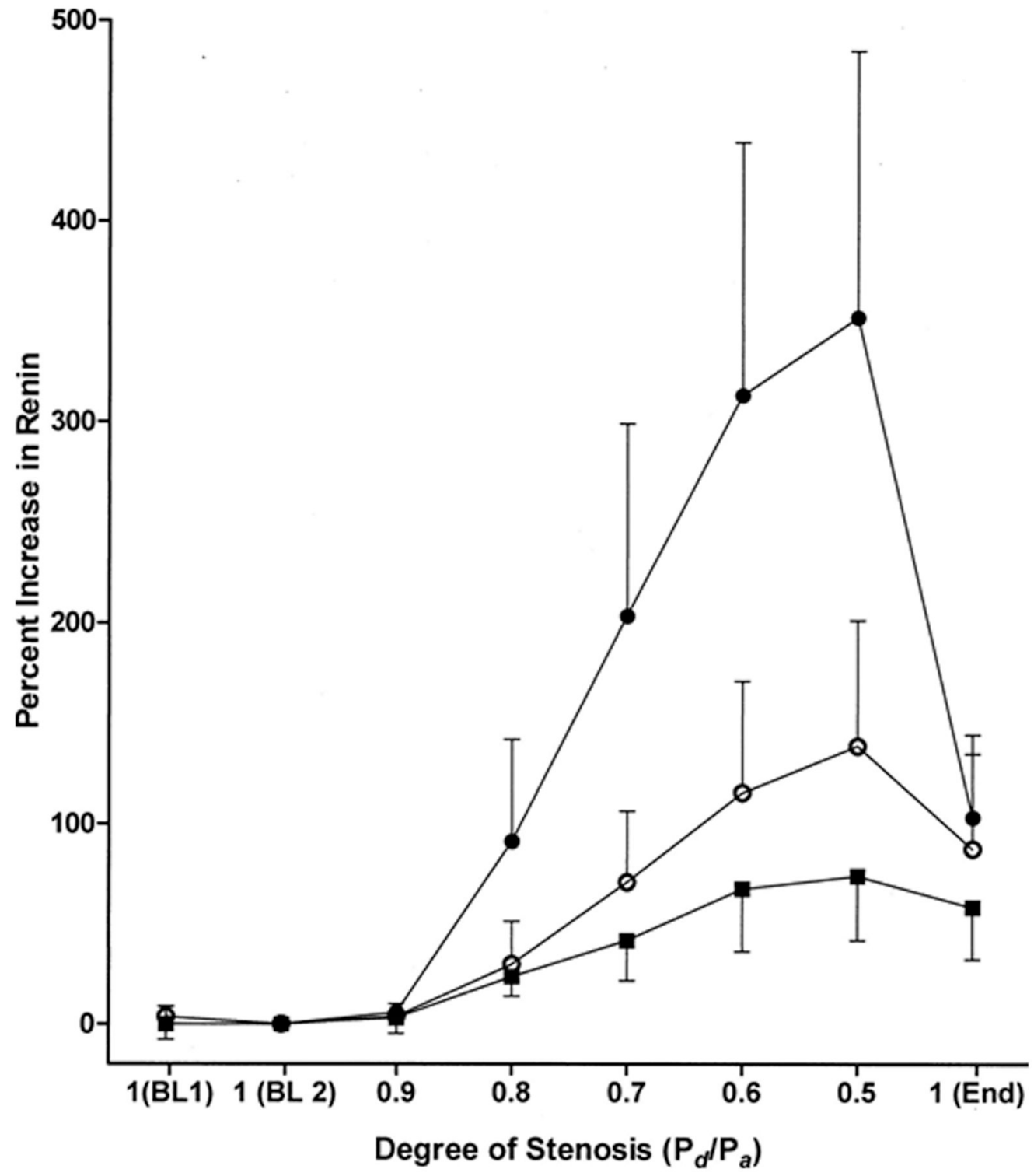

Figure 2:

Studies from human subjects with translesional pressure gradients indicate that an aorticrenal pressure gradient of $10 \%$ to $20 \%$ is necessary to detect renin release. Mean aortic pressure (Pd); mean pressure distal to the renal artery stenosis (Pa). (From De Bruyne B, Manoharan G, Pijls NHJ, et al. Assessment of renal artery stenosis severity by pressure gradient measurements. J Am Coll Cardiol. 2006; 48:1851-1855) 


\section{BOLD-MRI in Renovascular Disease}
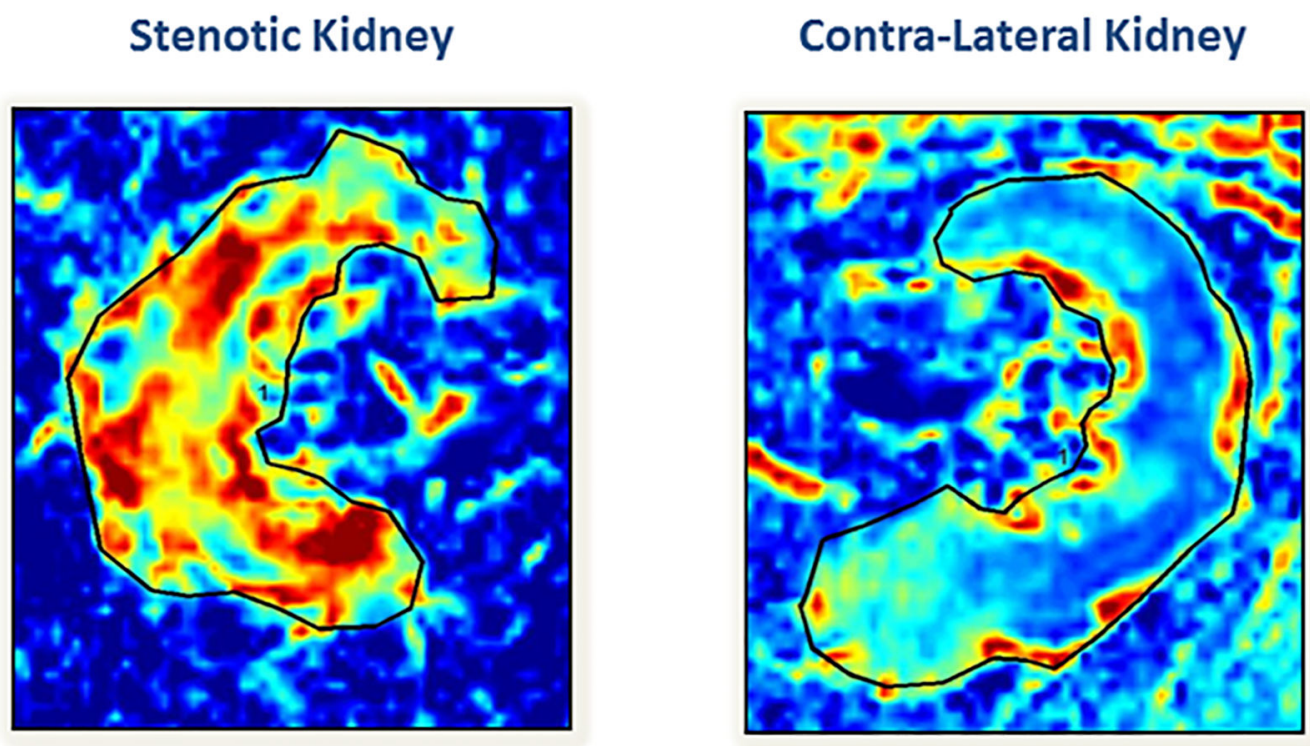

Low $\mathrm{O}_{2}$

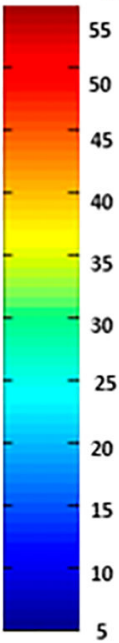

High $\mathrm{O}_{2}$

Figure 3:

Oxygenation is threatened lower in the post-stenotic kidney as compared to the nonstenotic contralateral kidney: Representative coronal blood oxygen level-dependent images. The R2* map (reflecting the level of deoxyhemoglobin) of the stenotic kidney shows a hypoxic cortical zone and widespread areas of elevated deoxyhemoglobin in the medullary segments (red). The unaffected contralateral kidney depicts the R2* map with a lower (blue) cortical zone and more gradual development of deeper medullary areas of hypoxia, and it is close to the appearance of a normal nonatherosclerotic renal artery stenosis kidney.(From Herrmann SM, Saad A, Eirin A, et al. Differences in GFR and Tissue Oxygenation, and Interactions between Stenotic and Contralateral Kidneys in Unilateral Atherosclerotic Renovascular Disease. Clin J Am Soc Nephrol. 2016 Mar 7; 11(3):458-69) 


\section{Spectrum of Renovascular Disease} Manifestations
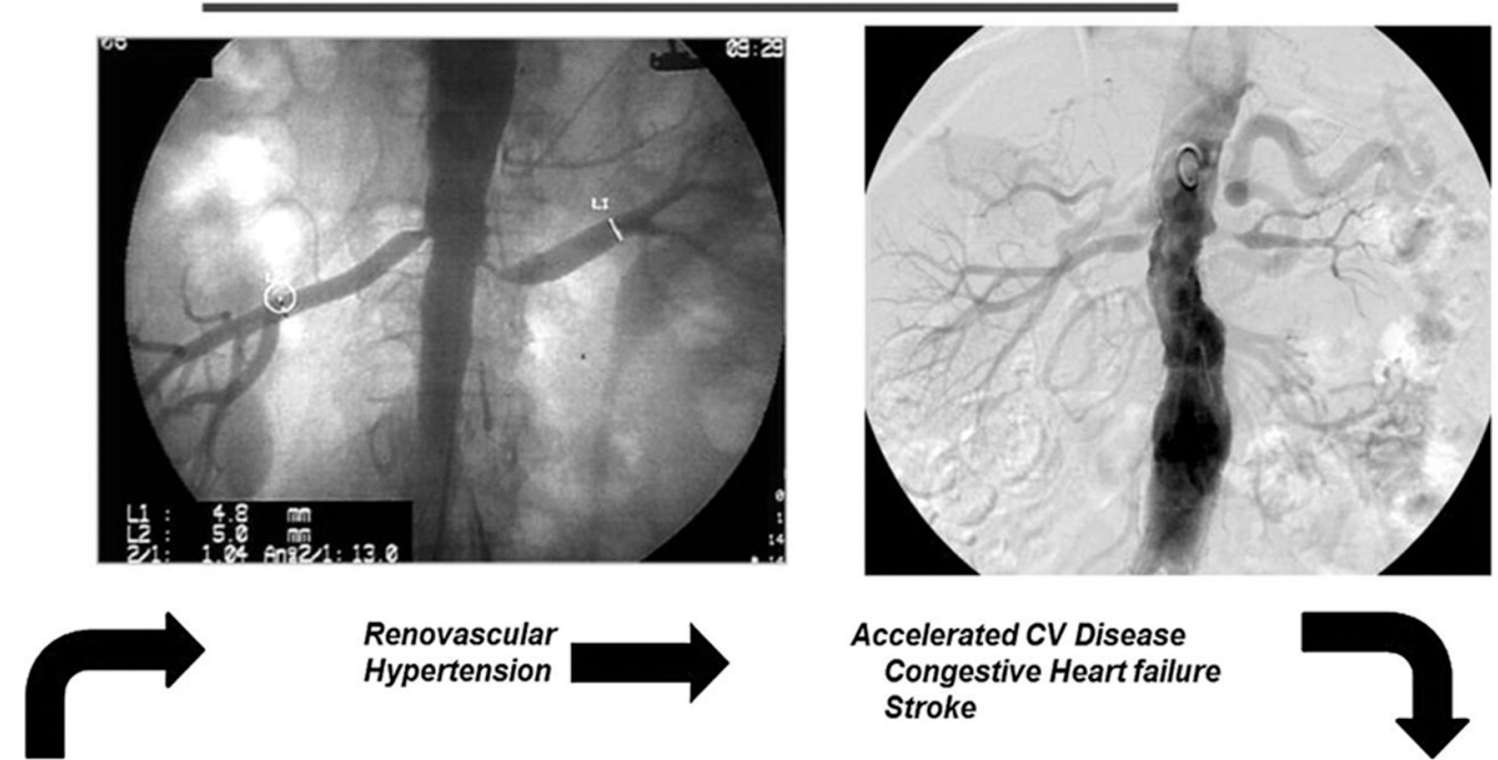

Renovascular Hypertension

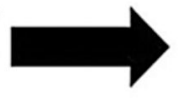

\section{Accelerated CV Disease Congestive Heart failure Stroke}

Asymptomatic

"Incidental RAS"

\section{Ischemic Nephropathy}

Figure 4:

Spectrum of atherosclerotic renovascular disease: Left panel, Aortogram obtained during coronary angiography demonstrating moderate incidental stenosis of both renal arteries in a 67-year-old man with symptomatic coronary disease. Right panel, More severe occlusive disease observed in a 68-year-old woman presenting with severe hypertension and episodes of flash pulmonary edema. Different clinical manifestations depending on severity of vascular occlusion, from asymptomatic hypertension to ischemic nephropathy. As occlusion progresses this leads to accelerated hypertension, circulatory congestion, and ultimately threatens viability of the kidney. (From Herrmann SM, Saad A, Textor SC. Management of Atherosclerotic Renovascular Disease after Cardiovascular Outcomes in Renal Atherosclerotic Lesions (CORAL). Nephrol Dial Transplant. 2015 Mar;30(3):366-75.) 
Hypertension \pm Reduced GFR

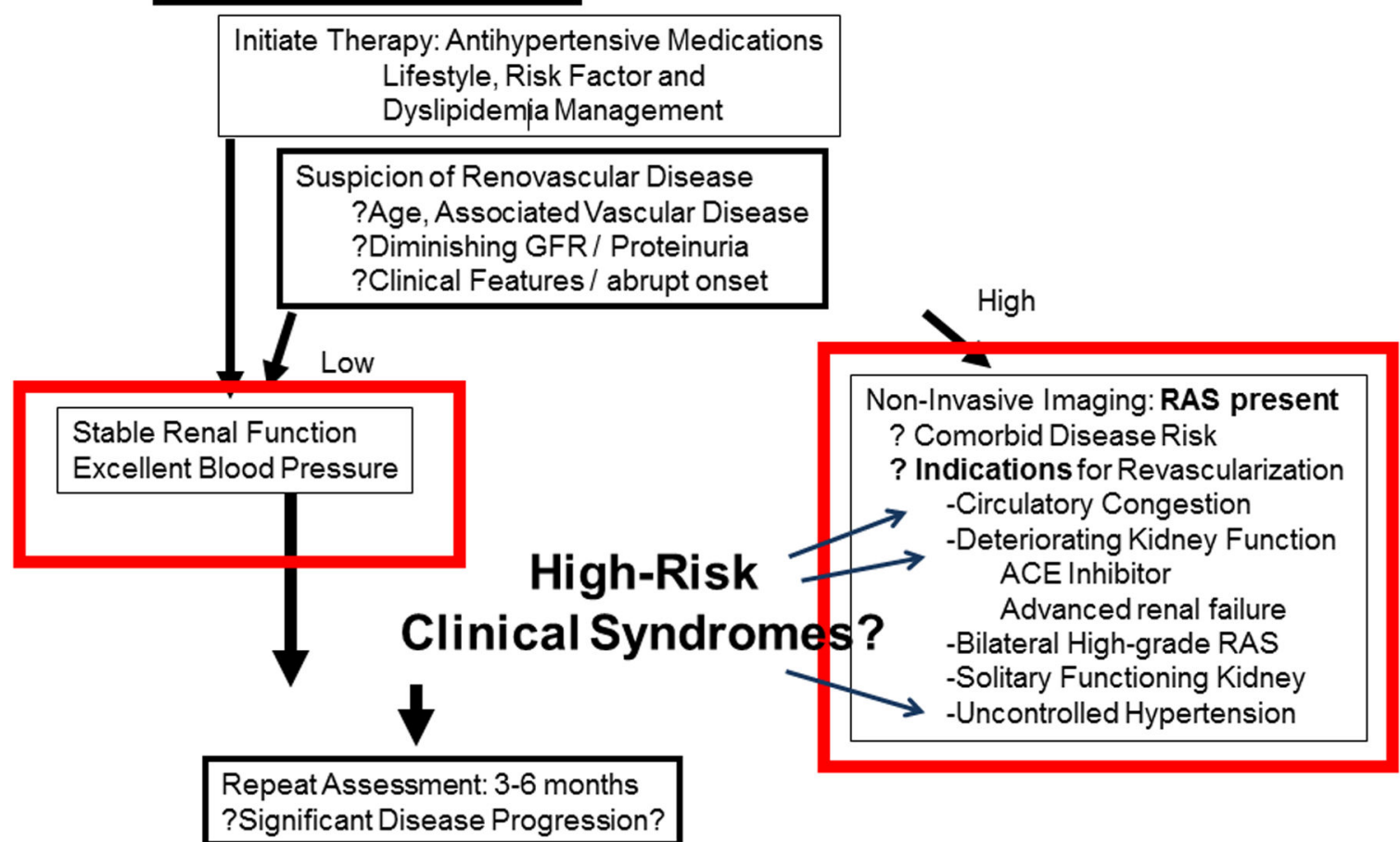

Figure 5:

Management of RVH and ischemic nephropathy. The goal is to reduce morbidity associated with hypertension by controlling blood pressure and preserving kidney function. If medical therapy fails or renovascular disease progresses, revascularization of renal artery should be considered. (From Herrmann SM, Saad A, Textor SC. Management of atherosclerotic renovascular disease after Cardiovascular Outcomes in Renal Atherosclerotic Lesions (CORAL). Nephrol Dial Transplant. 2015 Mar;30(3):366-75) 
Table 1:

Causes of Renovascular Disease. Data from 2005 American College of Cardiology/American Heart Association guidelines.

\begin{tabular}{l} 
Causes of Renovascular Diseases \\
Unilateral renal artery disease \\
Unilateral atherosclerotic renal artery stenosis \\
Unilateral fibromuscular dysplasias \\
Renal artery aneurism \\
Arterial embolus \\
Arteriovenous fistula (e.g., congenital, traumatic) \\
Segmental arterial occlusion (e.g., posttraumatic, radiation, thrombi) \\
Extrinsic compression of renal artery (e.g., tumor) \\
Bilateral renal artery disease or solitary functional kidney \\
Renal artery stenosis to a solitary kidney \\
Bilateral renal artery stenosis \\
Aortic coarctation \\
Atheroembolic disease \\
Systemic vasculitis (e.g., polyarteritis nodosa, Takayasu's arteritis) \\
Vascular occlusion due to endovascular stent graft \\
\hline
\end{tabular}


Table 2:

Clinical findings associated with renovascular disease: Data from 2005 American College of Cardiology/ American Heart Association guidelines. (Hirsch AT, Haskal ZJ, Hertzer NR, et al. ACC/AHA 2005 Practice Guidelines for the management of patients with peripheral arterial disease (lower extremity, renal, mesenteric, and abdominal aortic): a collaborative report from the American Association for Vascular Surgery/Society for Vascular Surgery, Society for Cardiovascular Angiography and Interventions, Society for Vascular Medicine and Biology, Society of Interventional Radiology, and the ACC/AHA Task Force on Practice Guidelines (Writing Committee to Develop Guidelines for the Management of Patients With Peripheral Arterial Disease): endorsed by the American Association of Cardiovascular and Pulmonary Rehabilitation; National Heart, Lung, and Blood Institute; Society for Vascular Nursing; TransAtlantic Inter-Society Consensus; and Vascular Disease Foundation. Circulation 2006;113:e463-654.)

\footnotetext{
Clinical Findings Associated with Renovascular Disease

Onset hypertension before age of 30 years old

Accelerated, resistant, malignant hypertension

Deterioration of renal function in response to angiotensin- converting enzyme inhibitors or angiotensin-receptor blocker

New onset of hypertension after 50 years of age (suggestive of atherosclerotic renal artery stenosis)

Asymmetric kidneys with more than $1.5 \mathrm{~cm}$ of difference in the size and otherwise unexplained loss of kidney function

Sudden unexplained pulmonary edema
} 